\title{
Ergodic statistical models: Entropic dynamics and chaos
}

Ignacio S. Gomez, and M. Portesi

Citation: AIP Conference Proceedings 1853, 100001 (2017); doi: 10.1063/1.4985374

View online: https://doi.org/10.1063/1.4985374

View Table of Contents: http://aip.scitation.org/toc/apc/1853/1

Published by the American Institute of Physics

\section{Articles you may be interested in}

Maximum entropy PDF projection: A review

AIP Conference Proceedings 1853, 070001 (2017); 10.1063/1.4985362

Jarzynski equality in the context of maximum path entropy

AIP Conference Proceedings 1853, 080003 (2017); 10.1063/1.4985368

New prior sampling methods for nested sampling - Development and testing

AIP Conference Proceedings 1853, 110003 (2017); 10.1063/1.4985378

Relating probability distributions using geodesic least squares regression: Application to edge-localized modes in fusion plasmas

AIP Conference Proceedings 1853, 100002 (2017); 10.1063/1.4985375

Probabilistic numerical methods for PDE-constrained Bayesian inverse problems

AIP Conference Proceedings 1853, 060001 (2017); 10.1063/1.4985359

Empirical study of Thompson sampling: Tuning the posterior parameters

AIP Conference Proceedings 1853, 050001 (2017); 10.1063/1.4985354

\section{Conference Proceedings}

\section{Get $30 \%$ off all \\ Enter Promotion Code PDF30 at checkout print proceedings!}




\title{
Ergodic statistical models: entropic dynamics and chaos
}

\author{
Ignacio S. Gomez ${ }^{1, a)}$ and M. Portesi ${ }^{1, b)}$ \\ ${ }^{1}$ IFLP, UNLP, CONICET, Facultad de Ciencias Exactas, Calle 115 y 49, 1900 La Plata, Argentina \\ a)nachosky@fisica.unlp.edu.ar \\ b) portesi@fisica.unlp.edu.ar
}

\begin{abstract}
We present an extension of the ergodic, mixing and Bernoulli levels of the ergodic hierarchy in dynamical systems, the information geometric ergodic hierarchy, making use of statistical models on curved manifolds in the context of information geometry. We discuss the $2 \times 2$ Gaussian Orthogonal Ensembles (GOE) within a $2 D$ correlated model. For values of the correlation coefficient vanishingly small, we find that GOE belong to the information geometric (IG) mixing level having a maximum negative value of scalar curvature. Moreover, we propose a measure of distinguishability for the family of distributions of the $2 D$ correlated model that results to be an upper bound of the IG correlation.
\end{abstract}

\section{INTRODUCTION}

Historically, several fundamental disciplines like statistical mechanics and thermodynamics in physics, have turned out to be adequately treated as theories of inference where the conclusions are derived from available information about the system and making use of a method of reasoning that involves probability theory. In this sense, one of the most important methods of inference is the maximum entropy method (ME method) where a rule is given for obtaining the distribution $p$ that represents the best knowledge of the system and corresponds to maximum uncertainty, as measured by a functional $S[p]$, constrained by the available information [1]. In particular, the so-called MaxEnt method results when the entropy $S[p]$ is chosen to be the Shannon-Gibbs one.

Given a well-established rule of inference like MaxEnt and generally other ME methods [2], one can study the dynamics of the system within that framework. The approach proposed by Caticha et al. known as Entropic Dynamics (ED) is to consider that the system moves irreversibly and continuously along the entropy gradient in a curved statistical manifold whose elements are probability distributions. There are several forms of ED depending on the variables and constraints that one chooses $[3,4,5]$. Curved statistical manifolds are the subject of study of the information geometry ( $\mathrm{IG})$, and they have associated the Fisher-Rao metric [6] which in turn is linked to the concepts of entropy and Fisher information [6,7]. Thus ED is a theoretical framework that arises from the combination of ME methods and the IG with the particularity of characterizing a system in terms of geometric quantities like the Ricci scalar and local scalar curvatures. It has proven to be useful in many applications $[8,5]$ and also, to study geometric phase transitions [9]. Furthermore using ED, asymptotic expressions for information measures are obtained by means of geodesic equations [5]. In this geometric framework, a criterion for characterizing global chaos can be obtained: the more negative is the curvature, the more chaotic is the dynamics. As usual, chaos can be characterized in terms of diverging initially nearby trajectories [10]. For the statistical models this condition results in the divergence of geodesic paths on the statistical manifold and constitutes a local criterion for chaos.

Besides, in dynamical systems theory, the ergodic hierarchy (EH) characterizes the chaotic behavior in terms of a type of correlation between subsets of the phase space [11,12]. In the asymptotic limit of large times, the EH establishes that the dynamics is more chaotic when the correlation decays faster. According to correlation decay, the four levels of EH are, from the weakest to the strongest: ergodic, mixing, Kolmogorov, and Bernoulli. In particular, in mixing systems any two subsets enough separated in time can be considered as "statistically independent" which allows one to use a statistical description of the behavior of the system. In the context of quantum chaos, the statistical independence is present in the universal statistical properties of energy levels which are given by the Gaussian ensembles [13, pp. 60-79]. In Gaussian ensembles theory one assumes that in a fully chaotic quantum system the in-

Bayesian Inference and Maximum Entropy Methods in Science and Engineering AIP Conf. Proc. 1853, 100001-1-100001-7; doi: 10.1063/1.4985374 Published by AIP Publishing. 978-0-7354-1527-0/\$30.00 
teractions are neglected in such way that the Hamiltonian matrix elements can be considered statistically independent [14]. Related to this, in $[15,16]$ a quantum extension of the EH was proposed, called the quantum ergodic hierarchy, which allowed to provide a characterization of the chaotic behaviors of the Casati-Prosen model [17] and the kicked rotator [13, pp. 145-150].

Inspired by the characterizations of quantum chaotic systems made in $[15,16]$ we discuss the $2 \times 2$ Gaussian Orthogonal Ensembles (GOE) within a $2 D$ correlated statistical model, and we propose a generalization of the ergodic, mixing and Bernoulli levels of the EH (for the sake of simplicity, in this contribution we do not deal with the Kolmogorov level which involves a $\sigma$-algebra) in the context of the IG and ED, called the information geometric ergodic hierarchy (IGEH). In order to study the correlation decay within IGEH we also define a distinguishability measure for a $2 D$ correlated model that allows us to give an upper bound for the information geometric correlation.

In this way, our main contribution is the information geometric ergodic hierarchy as an alternative framework for studying the chaotic dynamics in curved statistical models. The present work is organized as follows. First, we describe the GOE in terms of a $2 D$ correlated model, including a brief discussion about the dynamics characterized by the global chaos criteria. Second, we propose an information geometric extension of the ergodic hierarchy by expressing the correlations in terms of probability distributions instead of subsets of phase space. Next, we define a distinguishability measure for the $2 D$ correlated model, and an upper bound for the IGEH correlation is given. Finally, we draw some conclusions, and future research directions are outlined.

\section{GAUSSIAN ORTHOGONAL ENSEMBLES WITHIN A CURVED STATISTICAL MODEL}

In Gaussian Orthogonal Ensembles theory one deals with the probability distribution $p\left(H_{11}, H_{12}, \ldots, H_{N N}\right)$ for the Hamiltonian matrix elements assuming that the $H_{i j}$ are uncorrelated. Then in the framework of information geometry one could try to describe them by defining an appropriate microspace $\left\{\left(x_{1}, x_{2}, \ldots, x_{N}\right)\right\}$ that is the set of variables of the system and, a macrospace $\left\{\left(\theta_{1}, \theta_{2}, \ldots, \theta_{M}\right)\right\}$ which represents macroscopic quantities that can be measured in an experiment, like the mean value of any variable $\left\langle x_{j}\right\rangle$, etc.

In order to characterize the GOE within a statistical model we study a correlated ensemble of $2 \times 2$ matrices. We take the microspace as the Hamiltonian matrix elements $\left\{H_{11}, H_{22}, H_{12}, H_{21}\right\}$ and define the macrospace as follows. For the sake of simplicity, we choose the macrospace in such way that only $H_{11}$ and $H_{22}$ are correlated, and that the mean values of all variables are zero, except for the mean value corresponding to $H_{11}$ which is equal to $\mu$. Also, we consider that the variance of $H_{11}, H_{12}$ and $H_{21}$ are the same, denoted by $\sigma$. Moreover, in order to study how independent the diagonal Hamiltonian elements are, we restrict the dynamics by considering that $r \in[-1,1]$ is the correlation coefficient between $H_{11}$ and $H_{22}$, and that the product of the covariances between $H_{11}$ and $H_{22}$ is a constant $\Sigma^{2}$. Taking this into account, the resulting macrospace is $\{(\mu, \sigma) \in \mathbb{R} \times(0, \infty)\}$ where the constraints over the variables $\operatorname{are}^{1}$

$$
\begin{gathered}
\int p\left(H_{11}, H_{22}, H_{12}, H_{21}\right) H_{11} d H_{11} d H_{22} d H_{12} d H_{21}=\mu \\
\int p\left(H_{11}, H_{22}, H_{12}, H_{21}\right) H_{12} d H_{11} d H_{22} d H_{12} d H_{21}=\int p\left(H_{11}, H_{22}, H_{12}, H_{21}\right) H_{21} d H_{11} d H_{12} d H_{21} d H_{22}= \\
=\int p\left(H_{11}, H_{22}, H_{12}, H_{21}\right) H_{22} d H_{11} d H_{22} d H_{12} d H_{21}=0 \\
\int p\left(H_{11}, H_{22}, H_{12}, H_{21}\right)\left(H_{11}-\mu_{11}\right)^{2} d H_{11} d H_{22} d H_{12} d H_{21}=\int p\left(H_{11}, H_{22}, H_{12}, H_{21}\right) H_{12}^{2} d H_{11} d H_{22} d H_{12} d H_{21}= \\
=\int p\left(H_{11}, H_{22}, H_{12}, H_{21}\right) H_{21}^{2} d H_{11} d H_{22} d H_{12} d H_{21}=\sigma^{2} \\
\int p\left(H_{11}, H_{22}, H_{12}, H_{21}\right) H_{22}^{2} d H_{11} d H_{22} d H_{12} d H_{21}=\Sigma^{4} / \sigma^{2}, \quad \Sigma=\text { constant } \\
\int p\left(H_{11}, H_{22}, H_{12}, H_{21}\right)\left(H_{11}-\mu\right) H_{22} d H_{11} d H_{22} d H_{12} d H_{21}=r \Sigma^{2} \quad, \quad-1 \leq r \leq 1
\end{gathered}
$$

\footnotetext{
${ }^{1}$ Note that, since the GOE correspond to the orthogonal class of Hamiltonians then one has that $H_{12}=H_{21}$. However, in the formalism of Random matrices and for the orthogonal case, the volume element $d H_{11} d H_{22} d H_{12} d H_{21}$ (as if $H_{12}$ and $H_{21}$ were independent variables) is the real Lebesgue measure of $\mathbb{R}^{4}$ and must to be taken into account in order to normalize the probability distribution [13].
} 
with the normalization condition

$$
\int p\left(H_{11}, H_{22}, H_{12}, H_{21}\right) d H_{11} d H_{22} d H_{12} d H_{21}=1
$$

According to MaxEnt, the probability distribution $p\left(H_{11}, H_{22}, H_{12}, H_{21} \mid \mu, \sigma ; r\right)$ that maximizes the Shannon-Gibbs entropy subjected to (1) and (2) is [5]

$$
\begin{gathered}
p\left(H_{11}, H_{22}, H_{12}, H_{21} \mid \mu, \sigma ; r\right)= \\
\frac{1}{2 \pi \Sigma^{2} \sqrt{1-r^{2}}} \exp \left(-\frac{1}{2\left(1-r^{2}\right)}\left[\frac{1}{\sigma^{2}}\left(H_{11}-\mu\right)^{2}+\frac{\sigma^{2}}{\Sigma^{4}} H_{22}^{2}-2 r \frac{1}{\Sigma^{2}}\left(H_{11}-\mu\right) H_{22}\right]\right) \frac{1}{2 \pi \sigma^{2}} \exp \left(-\frac{1}{2 \sigma^{2}}\left(H_{12}^{2}+H_{21}^{2}\right)\right)
\end{gathered}
$$

where the correlation coefficient $r$ is considered as an external parameter (and therefore, not a macrovariable) and since the correlation between $H_{11}$ and $H_{22}$ is in terms of $r$, due to Eq. (1), then $\Sigma$ can be taken as a fixed constant. Therefore, only $\mu$ and $\sigma$ are the macrovariables of the macrospace. The Fisher-Rao metric for this model is to be computed as

$$
\begin{gathered}
g_{i j}=\int d H_{11} d H_{22} d H_{12} d H_{21} p\left(H_{11}, H_{22}, H_{12}, H_{21} \mid \mu, \sigma ; r\right) \frac{\partial \log p\left(H_{11}, H_{22}, H_{12}, H_{21} \mid \mu, \sigma ; r\right)}{\partial \theta_{i}} \frac{\partial \log p\left(H_{11}, H_{22}, H_{12}, H_{21} \mid \mu, \sigma ; r\right)}{\partial \theta_{j}} \\
\text { with } i, j=1,2 \text { and } \theta_{1}=\mu, \theta_{2}=\sigma
\end{gathered}
$$

where the integration is taken over all the microspace $\left\{\left(H_{11}, H_{22}, H_{12}, H_{21}\right) \in \mathbb{R}^{4}\right\}$. With the help of [5] one obtains

$$
g_{11}=\frac{1}{\sigma\left(1-r^{2}\right)} \quad, \quad g_{22}=\frac{4}{\sigma\left(1-r^{2}\right)} \quad, \quad g_{12}=g_{21}=0
$$

From this, one can obtain the Christoffel symbols $\Gamma_{i j}^{k}$ whose nonvanishing coefficients are

$$
\Gamma_{12}^{1}=\Gamma_{21}^{1}=-\frac{1}{\sigma} \quad, \quad \Gamma_{11}^{2}=\frac{1}{4 \sigma} \quad, \quad \Gamma_{22}^{2}=-\frac{1}{\sigma}
$$

Using (5) and (6) one can calculate the non vanishing components of the Ricci tensor $R_{i j}$ and the Ricci scalar curvature $R$, thus giving

$$
R=g^{11} R_{11}+g^{22} R_{22}=-\frac{1}{2}\left(1-r^{2}\right) \quad \text {, with } \quad R_{11}=-\frac{1}{4 \sigma^{2}}, R_{22}=-\frac{1}{\sigma^{2}}
$$

Three remarks follow. First, the statistical manifold has a curvature which is negative for all values of the correlation coefficient $r \in[-1,1]$. Based on the global chaos criterion above, this simply means that the dynamics in macrospace $(\mu, \sigma)$ is chaotic for all $r$.

Second, the $2 \times 2 \mathrm{GOE}$ case corresponds to $r=0$ and $\Sigma=\sigma$, thus having the minimum value of the scalar curvature

$$
R_{G O E}=R(r=0)=-\frac{1}{2}=R_{\min } \quad \text { (GOE, most chaotic case) }
$$

Third, for the strongly correlated case that corresponds to $|r| \sim 1$ one has

$$
R(|r| \rightarrow 1)=0 \quad \text { (strongly correlated case) }
$$

which can be interpreted, by the global chaos criterium, as the case when the dynamics is the least chaotic of all.

\section{TOWARDS AN INFORMATION GEOMETRIC DEFINITION OF THE ERGODIC HIERARCHY}

Motivated by the characterization of chaotic dynamics made in $[15,16]$ by means of a quantum extension of the ergodic hierarchy, now we study a generalization of the EH in the context of the information geometry. This allows one to measure how independent the variables $H_{i j}$ are for the $2 \times 2$ correlated ensemble (3). 
If one has a dynamical system $\left(X, \Sigma, \mu,\left\{T_{t}\right\}_{t \in J}\right)$ where $X$ is a set, $\Sigma$ is a sigma-algebra of $X, \mu$ a measure defined over $\Sigma$ and $\left\{T_{t}\right\}_{t \in J}$ a group of measure-preserving transformations, then the EH correlation $C\left(T_{t} A, B\right)$ between two subsets $A$ and $B$ of $X$ that are separated by a time $t$ is

$$
C\left(T_{t} A, B\right)=\mu\left(T_{t} A \cap B\right)-\mu(A) \mu(B)
$$

The ergodic, mixing and Bernoulli levels of the EH are given in terms of (10) in the following way. Given two arbitrary sets $A, B \in X$, it is said that $T_{t}$ is

- ergodic if

$$
\lim _{T \rightarrow \infty} \frac{1}{T} \int_{0}^{T} C\left(T_{t} A, B\right) d t=0
$$

- mixing if

$$
\lim _{t \rightarrow \infty} C\left(T_{t} A, B\right)=0
$$

- Bernoulli if

$$
C\left(T_{t} A, B\right)=0 \quad \text { for all } t \geq 0
$$

In ergodic systems the correlation vanishes "in time average" for large times while in mixing systems $C\left(T_{t} A, B\right)$ vanishes for $t \rightarrow \infty$. In Bernoulli systems the correlation is zero for all times. These levels classify the dynamics in terms of the type of decay of $C\left(T_{t} A, B\right)$ according to Eqs. (11), (12) and (13).

In order to express $C\left(T_{t} A, B\right)$ by means of probability distributions it is is more convenient to use the definition of (10) in terms of distribution functions, which is given by

$$
C\left(f \circ T_{t}, g\right)=\int_{X}\left(f \circ T_{t}\right)(x) g(x) d x-\int_{X} f(x) d x \int_{X} g(x) d x \quad \forall f, g \in \mathbb{L}^{1}(X)
$$

where $f \circ T_{t}$ denotes the composition of $f$ and $T_{t}$, i.e. $f \circ T_{t}(x)=f\left(T_{t}(x)\right)$ for all $x \in X$ and now the role of $A, B$ is played by the functions $f, g \in \mathbb{L}^{1}(X)$. Physically, $f$ represents any initial density function of the classical system whose value at time $t$ is given by $f \circ T_{t}$, and where $T_{t}$ is (in Hamiltonian systems) the classical Liouville evolution.

Now, in information geometry and entropic dynamics one has probability distributions $p(x \mid \theta)$ that depend on a set of parameters $\theta$, and the dynamics of the macrovariables $\theta$ is along the geodesics of the statistical manifold. Moreover, in the statistical manifold the role of time variable $t$ of dynamical systems is played by a parameter $\tau$ along the geodesics. In order to introduce information geometry methods we propose the following approach by defining a correlation between functions as the macrovariables $\theta$ evolve along the geodesics. Given $N$ functions $f\left(x_{i}\right)$, each one of them in terms of the variable $x_{i}$ for all $i=1, \ldots, N$, we propose the following $I G$ correlation $C\left(f_{1}, \ldots, f_{N}, \tau\right)$ between $f_{1}, \ldots, f_{N}$ at time-like parameter $\tau$ as

$$
\begin{gathered}
C\left(f_{1}, \ldots, f_{N}, \tau\right) \doteq \\
\int p\left(x_{1}, \ldots, x_{l} \mid \theta(\tau)\right) \prod_{i=1}^{N} f_{i}\left(x_{i}\right) d x_{1} \cdots d x_{l}-\prod_{i=1}^{N} \int p_{i}\left(x_{i} \mid \theta(\tau)\right) f_{i}\left(x_{i}\right) d x_{i}
\end{gathered}
$$

where $\theta(\tau)=\left(\theta_{1}(\tau), \ldots, \theta_{M}(\tau)\right)$ is the M-dimensional vector of the macrovariables at "time" $\tau$ and,

$$
p_{i}\left(x_{i} \mid \theta(\tau)\right)=\int p\left(x_{1}, \ldots, x_{N} \mid \theta(\tau)\right) \prod_{j \neq i} d x_{j} \quad, \quad i=1 \ldots N
$$

are the marginal distributions of $p\left(x_{1}, \ldots, x_{N} \mid \theta(\tau)\right)$. From (15) we can see that $C\left(f_{1}, \ldots, f_{N}, \tau\right)$ measures how independent the variables $x_{1}, \ldots, x_{N}$ are at $\tau$, and this can be considered as a sort of information geometric generalization of the EH correlation.

Having established $C\left(f_{1}, \ldots, f_{N}, \tau\right)$ and taking into account the ergodic, mixing and Bernoulli levels given by Eqs. (11), (12) and (13), we define the information geometric ergodic hierarchy (IGEH) as follows. Given a set of $N$ arbitrary functions $f_{1}\left(x_{1}\right), \ldots, f_{N}\left(x_{N}\right)$ we say that now the statistical model is 
- IG ergodic if

$$
\lim _{T \rightarrow \infty} \frac{1}{T} \int_{0}^{T} C\left(f_{1}, \ldots, f_{N}, \tau\right) d \tau=0
$$

- IG mixing if

$$
\lim _{\tau \rightarrow \infty} C\left(f_{1}, \ldots, f_{N}, \tau\right)=0
$$

- IG Bernoulli if

$$
C\left(f_{1}, \ldots, f_{N}, \tau\right)=0 \quad \text { for all } t \in \mathbb{R}
$$

As an example, taking $r=0$ and $\Sigma=\sigma$ in (3) we obtain the $2 \times 2$ GOE probability distribution which is simply the product of its marginal distributions. Then from (15) and (19) it follows that the $2 \times 2 \mathrm{GOE}$ is a statistical model that is IG Bernoulli.

A statistical model that is IG ergodic can be given by assuming that $C\left(f_{1}, \ldots, f_{N}, \tau\right)$ is, for instance, proportional to $\sin (\alpha \tau)\left\|f_{1}\right\|_{1} \ldots\left\|f_{N}\right\|_{1}$ with $\alpha \in \mathbb{R}$. Making this replacement in (17) one obtains that $\lim _{T \rightarrow \infty} \frac{1}{T} \int_{0}^{T} C\left(f_{1}, \ldots, f_{N}, \tau\right) d \tau=0$. Since $\sin (\alpha \tau)\left\|f_{1}\right\|_{1} \ldots\left\|f_{N}\right\|_{1}$ oscillates, then this model is not IG mixing nor IG Bernoulli.

Our approach, thus, deals with ergodic hierarchy in statistical models from an information geometry viewpoint. Henceforth, we use ergodic statistical model to designate any statistical model satisfying (17), (18) or (19).

\section{A DISTINGUISHABILITY MEASURE FOR THE 2D CORRELATED MODEL}

In order to use the levels of the IGEH to characterize the dynamics of statistical models one should have a manner of determining the decay of correlation $C\left(f_{1}, \ldots, f_{N}, \tau\right)$ in Eqs. (17), (18) or (19). For the family of the $2 D$ correlated probabilities $p\left(H_{11}, H_{22}, H_{12}, H_{21} \mid \mu, \sigma ; r\right)$ of Eq. (3) we define the distinguishability measure $F:\left\{p\left(H_{11}, H_{22}, H_{12}, H_{21} \mid \mu, \sigma ; r\right) \mid \mu \in(-\infty, \infty), \sigma \in(0, \infty),-1 \leq r \leq 1\right\} \longmapsto \mathbb{R}$, given by

$$
\begin{gathered}
F(p) \doteq\left\|p\left(H_{11}, H_{22}, H_{12}, H_{21} \mid \mu, \sigma ; r\right)-p\left(H_{11}\right) p\left(H_{22}\right) p\left(H_{12}\right) p\left(H_{21}\right)\right\|_{\infty} \\
=\max _{\left(H_{11}, H_{22}, H_{12}, H_{21}\right) \in \mathbb{R}^{4}}\left|p\left(H_{11}, H_{22}, H_{12}, H_{21} \mid \mu, \sigma ; r\right)-p\left(H_{11}\right) p\left(H_{22}\right) p\left(H_{12}\right) p\left(H_{21}\right)\right|
\end{gathered}
$$

where $p\left(H_{i j}\right)$ with $i, j=1,2$ are the marginal distributions of $p\left(H_{11}, H_{22}, H_{12}, H_{21} \mid \mu, \sigma ; r\right)$. Furthermore, if $f_{1}\left(H_{11}\right), f_{2}\left(H_{22}\right), f_{3}\left(H_{12}\right), f_{4}\left(H_{21}\right) \in \mathbb{L}^{1}(\mathbb{R})$ are arbitrary functions of $H_{11}, H_{22}, H_{12}, H_{21}$, then we have

$$
\begin{aligned}
& \left|C\left(f_{1}, f_{2}, f_{3}, f_{4}, \tau\right)\right| \\
& =\left|\int d^{4} H_{i j} p\left(H_{11}, H_{22}, H_{12}, H_{21} \mid \mu, \sigma ; r\right) f_{1}\left(H_{11}\right) f_{2}\left(H_{22}\right) f_{3}\left(H_{12}\right) f_{4}\left(H_{21}\right)-\left\langle f_{1}\left(H_{11}\right)\right\rangle\left\langle f_{2}\left(H_{22}\right)\right\rangle\left\langle f_{3}\left(H_{12}\right)\right\rangle\left\langle f_{4}\left(H_{21}\right)\right\rangle\right| \\
& \leq \max _{\left(H_{11}, H_{22}, H_{12}, H_{21}\right) \in \mathbb{R}^{4}}\left|p\left(H_{11}, H_{22}, H_{12}, H_{21} \mid \mu, \sigma, r\right)-p\left(H_{11}\right) p\left(H_{22}\right) p\left(H_{12}\right) p\left(H_{21}\right)\right| \\
& \times\left|\int d^{4} H_{i j} f_{1}\left(H_{11}\right) f_{2}\left(H_{22}\right) f_{3}\left(H_{12}\right) f_{4}\left(H_{21}\right)\right| \leq F(p)\left\|f_{1}\right\|_{\infty}\left\|f_{2}\right\|_{\infty}\left\|f_{3}\right\|_{\infty}\left\|f_{4}\right\|_{1}
\end{aligned}
$$

where $d^{4} H_{i j}$ is the volume element $d H_{11} d H_{22} d H_{12} d H_{21}$ and

$$
\begin{aligned}
\left\langle f_{1}\left(H_{11}\right)\right\rangle & =\int p\left(H_{11}\right) f_{1}\left(H_{11}\right) d H_{11} \\
\left\langle f_{2}\left(H_{22}\right)\right\rangle & =\int p\left(H_{22}\right) f_{2}\left(H_{22}\right) d H_{22} \\
\left\langle f_{3}\left(H_{12}\right)\right\rangle & =\int p\left(H_{12}\right) f_{3}\left(H_{12}\right) d H_{12} \\
\left\langle f_{4}\left(H_{21}\right)\right\rangle & =\int p\left(H_{21}\right) f_{4}\left(H_{21}\right) d H_{21}
\end{aligned}
$$

Eq. (21) expresses that $F(p)\left\|f_{1}\right\|_{\infty}\left\|f_{2}\right\|_{\infty}\left\|f_{3}\right\|_{\infty}\left\|f_{4}\right\|_{1}$ is un upper bound for $\left|C\left(f_{1}, f_{2}, f_{3}, f_{4}, \tau\right)\right|$. Therefore, it is convenient to find an analytic expression for (20). After some algebra one can obtain that

$$
F(p)=|r|\left(\sqrt{1-r^{2}}(1+|r|)\right)^{-1-\frac{1}{r}} \quad \text { for all } r \in[-1,1]
$$




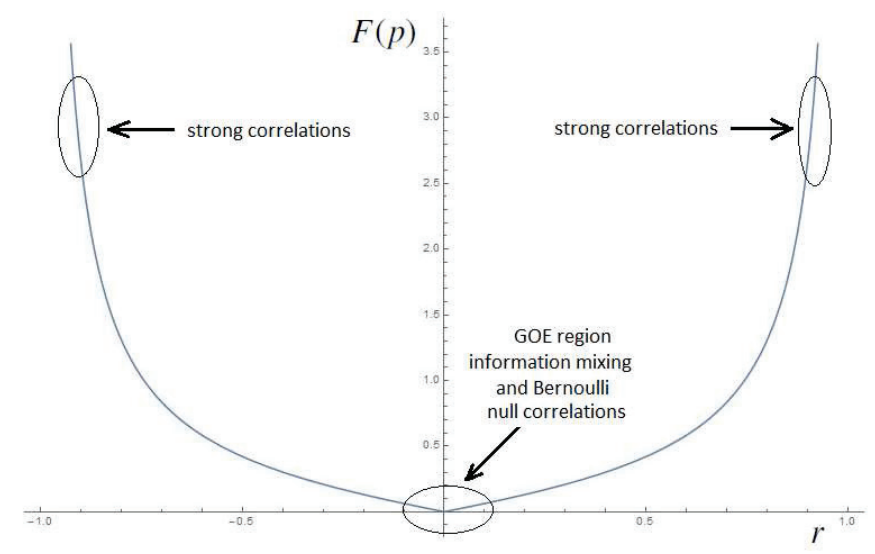

FIGURE 1. Behavior of $F(p)$ in terms of the correlation coefficient. The Gaussian Orthogonal Ensembles correspond to the region near $r=0$ where the statistical model belongs to the IG Bernoulli level. When $r \rightarrow \pm 1$ one has that $F(p)$ diverges with the presence of strong correlations. The discontinuity of the slope of $F$ at $r=0$ is due to the particular form of $F(p)$, i.e. the maximum operation of infinite norm can present discontinuities in its derivatives.

The behavior of $F(p)$ "which is independent of $\mu$ and $\sigma$ " is shown in Fig. 1. Two relevant regions, corresponding to the limiting cases $r \rightarrow 0$ and $r \rightarrow \pm 1$, can be well distinguished. The region $r \rightarrow 0$ corresponds to the zone near the GOE which is characterized by the IG mixing and IG Bernoulli levels, with the particularity that the variables of microspace are uncorrelated. Moreover, we can see that near to $r=0$ the decay is linear in $r$. The curve $F(p)$ also shows that, if $r \rightarrow 0$ when $\tau \rightarrow \infty$, then the statistical model is IG mixing.

In the region $r \rightarrow \pm 1$ the measure $F(p)$ diverges corresponding to the maximally correlated case, which physically means that the system presents strong correlations between the variables of microspace. Due to the correlations are strong in this regime the statistical model cannot be IG mixing nor IG Bernoulli.

Finally, it should be noted that $F(p)$ does not allow one to distinguish between two probability distributions having $r$ and $-r$ respectively. The symmetry respect to the axis $r=0$ is due to the mathematical form of the infinite norm $\|.\|_{\infty}$ in the definition (20). That is, with other choices of $F(p)$ one could distinguish states (probability distributions) with correlation coefficients $r$ and $-r$.

\section{CONCLUSIONS}

We proposed a generalization, called "the information geometric ergodic hierarchy (IGEH)", of the ergodic, mixing and Bernoulli levels in the context of information geometry and we applied it to characterize the $2 \times 2$ Gaussian Orthogonal Ensembles.

By defining a measure for the family of the $2 D$ correlated probability distributions we obtained an upper bound for the IG correlation which allowed one to give a sufficient condition for the IG mixing level when $\lim _{\tau \rightarrow \infty} r(\tau)=0$, $r$ being the correlation coefficient and $\tau$ the parameter along geodesics. In other words, since the correlation goes to zero for $r \rightarrow 0$ and this implies IG mixing as one can deduce from its definition (18) and Eq. (21), then the condition $\lim _{\tau \rightarrow \infty} r(\tau)=0$ is a sufficient condition for the IG mixing level.

The relevance of our main contribution, the information geometric ergodic hierarchy (IGEH), lies in the following remarks:

- The IGEH generalizes the notions of statistical independence and chaos characterization of the ergodic hierarchy to statistical models on curved manifolds in the context of the information geometry. In turn, this gives rise to an ergodic hierarchy characterization of statistical models, which we called ergodic statistical models.

- Geometrical notions and the global chaos criterion of entropic dynamics can be related with the levels of the IGEH. The $2 \times 2$ GOE case belonging to the most chaotic level, the IG Bernoulli, has an associated minimum negative value of the scalar curvature $R_{G O E}=-\frac{1}{2}$.

- By obtaining upper bounds $F(p)$ on the IG correlation for a specific family of probability distributions, as exemplified by the curve of Fig. 1, one could study geometrical phase transitions moving along curves $F(p)$ as an external parameter $r$ is varied. 


\section{ACKNOWLEDGMENTS}

This work was partially supported by CONICET (National Research Council) and Universidad Nacional de La Plata, La Plata, Argentina.

\section{REFERENCES}

[1] Jaynes, E. T. Information Theory and Statistical Mechanics, I, II. Phys. Rev. 1957, 106, 620-630; 108, 171-190.

[2] Giffin, A. Maximum Entropy: The Universal Method for Inference. PhD Thesis, State University of New York at Albany, USA, 2008.

[3] Caticha, A. Entropic dynamics, AIP Conf. Proc. 2002, 617, 302-313; Entropic Dynamics, Time and Quantum Theory. J. Phys. A, 2011, 44, 225303.

[4] Caticha, A., Giffin, A. Updating probabilities, AIP Conf. Proc. 2006, 872, 31-42.

[5] Cafaro, C. Works on an information geometrodynamical approach to chaos. Chaos Solitons and Fractals 2009, 41, 886-891.

[6] Rao, C. Differential Geometry in Statistical Inference. In chap. Differential metrics in probability spaces; Institute of Mathematical Statistics, Hayward, CA, 1987.

[7] Amari, S., Nagaoka, H. Methods of Information Geometry; Oxford University Press: Oxford, UK, 2000.

[8] Verdoolaege, G. Geodesic least squares regression for scaling studies in magnetic confinement fusion, AIP Conf. Proc. 2014, 1641, 564-571; Robust regression on noisy data for fusion scaling laws. Rev. Sci. Instrum. 2014, 85, 11E810.

[9] Portesi, M., Plastino, A., Pennini, F. Information measures based on Tsallis' entropy and geometric considerations for thermodynamic systems, Phys. A 2006, 365, 173-176; Geometrical aspects of a generalized statistical mechanics, Phys. A 2007, 373, 273-282.

[10] Lichtenberg A. J., Lieberman M. A., Regular and Chaotic Dynamics (Applied Mathematical Sciences), Springer, Berlin, 2010, p. 304.

[11] Berkovitz, J., Frigg, R., Kronz, F. The Ergodic Hierarchy, Randomness and Hamiltonian Chaos, Stud. Hist. Phil. Mod. Phys. 2006, 37, 661-691.

[12] Lasota, A., Mackey, M. In Probabilistic properties of deterministic systems; Cambridge Univ. Press, Cambridge, 1985, pp. 45-113.

[13] Stockmann, H. In Quantum Chaos - An Introduction; Cambridge Univ. Press, Cambridge, 1999.

[14] Bohigas, O., Giannoni, M., J., Schmit, C. Characterization of Chaotic Quantum Spectra and Universality of Level Fluctuation Laws, Phys. Rev. Lett. 1984, 52, 1.

[15] Gomez, I., Castagnino, M. Towards a definition of the Quantum Ergodic Hierarchy: Kolmogorov and Bernoulli systems, Phys. A 2014, 393, 112-131.

[16] Gomez, I., Losada, M., Fortin, S., Castagnino, M., Portesi, M. A Semiclassical Condition Based on Pesin Theorem. Int. Journ. Theor. Phys., 2015, 7, 2192-2203.

[17] Casati, G., Prosen, T. Quantum chaos and the double slit experiment, Phys. Lett. A 2005, 72, 032111. 